\title{
The COVID-19 pandemic and research shutdown: staying safe and productive
}

\author{
M. Bishr Omary, ${ }^{1}$ Jeetendra Eswaraka, ${ }^{2}$ S. David Kimball, ${ }^{2}$ Prabhas V. Moghe, ${ }^{3}$ Reynold A. Panettieri Jr., ${ }^{4}$ and Kathleen W. Scotto ${ }^{5}$ \\ ${ }^{1}$ Center for Advanced Biotechnology and Medicine, ${ }^{2}$ Office of Research and Economic Development, ${ }^{3}$ Department of Biomedical Engineering, ${ }^{4}$ Institute for Translational Medicine and Science, and ${ }^{5}$ Robert \\ Wood Johnson Medical School, Rutgers University, Piscataway, New Jersey, USA.
}

$\mathbf{T}_{\mathrm{he}}$ he coronavirus disease 2019 (COVID-19) pandemic, caused by SARS-CoV-2, has caused severe disease outcomes and widespread infections not seen in terms of its case fatality rate and global economic impact since the 1918 to 1919 Spanish flu, with the latter leading to 675,000 deaths in the United States (US) (50 million worldwide) (1). The US currently has the largest number of SARS-CoV-2 infections in the world, with tremendous health, economic, and social ramifications, including closing of schools, businesses, and all public gatherings in order to maximize social distancing and prevent virus spread. Here, we address the major impact of the COVID-19 pandemic on biomedical research, the challenges created by the COVID-19 pandemic for research-intensive institutions (Figure 1A), and what investigators can do to maintain some level of research activity while keeping their coworkers and trainees safe and engaged (Figure 1B).

\section{Biomedical research operations across US institutions}

In February 2020, the potential impact of COVID-19 in the US started to manifest as the case trajectory accelerated in Italy. Universities began to implement emergency operation plans, which rapidly escalated as SARS-CoV-2 began its exponential spread. Driven by both the imperative for social distancing and individual state executive orders, many research institutions have severely reduced onsite research to activities deemed essential (Supplemental Table 1; supplemental material available online with this article; https://doi.org/10.1172/ JCI138646DS1). At Rutgers University, we instituted policies similar to those of other major research institutions. The three principles that informed our decisions are (a) noncritical research and research-related activities must be performed remotely, (b) strict adherence to social distancing, and (c) new projects that require in-person presence are not permitted, excluding all COVID-19-related basic and clinical research, which are permitted and encouraged. "Dry" laboratory research is carried out remotely unless specific dispensation is warranted, and active therapeutic clinical trials remain ongoing. Research activities that are deemed critical are permitted, with one designated "critical laboratory member" permitted onsite at a time, with a possible rotation system if essential. Graduate students and postdoctoral fellows, but not undergraduate students, can be named critical personnel but only by mutual agreement between trainees and mentors.

While research unrelated to COVID-19 has slowed and remote work has placed significant constraints on the efficiency of operations, research on COVID-19 has exploded with energy and urgency. University researchers published and deposited in the Protein Data Bank the first structure of the SARS-CoV-2 main protease (2) only weeks after the disease erupted in China. Universities have created internal funding mechanisms for coronavirus research (e.g., Pennsylvania State University funded 17 proposals with $\$ 1.2$ million by March 27, 2020), and investigators quickly moved to deliver solutions to the personal protective equipment (PPE) crisis. Many laboratories have pivoted their research efforts to respond to the health care crisis. Some programs, like Rutgers University's Cell and DNA Repository (3), have
Conflict of interest: SDK has stock in Bristol-Myers Squibb, Lexicon Pharmaceuticals, and Merck as part of a 401k. RAP consults with AstraZeneca with more than $\$ 10,000$ in renumeration, and has grant funding from AstraZeneca, MedImmune, Research Institute for Fragrance Materials, Equillium, Genentech, OncoArendi, and Metera. Copyright: () 2020, American Society for Clinical Investigation.

Reference information: J Clin Invest. 2020;130(6):2745-2748. https://doi.org/10.1172/JCI138646. set up high-throughput COVID-19 detection facilities to test up to 10,000 samples daily. Engineers at the University of Wisconsin collaborated with local industry to design and provide open-source plans for manufacturing face shields (4). The power of basic biomedical research to continue delivering solutions to the COVID-19 pandemic are exemplified by Cepheid's Emergency Use Authorization for a 45-minute point-of-care (POC) test (5), and Abbott Laboratories' announcement of a less than 15-minute POC test (6). The challenge of quickly implementing these discoveries and products in the fight against COVID-19 remains a logistical and political one, requiring universities to work closely with private and government agencies.

The COVID-19 pandemic has also impacted research administration. The urgency for high quality and rapid turnover of COVID-19-related studies from the IRB, IACUC, COI, biosafety, and contracting functions in our institution and others (7) has transformed the workings of these key regulatory functions. The potential for repurposing marketed drugs to treat COVID-19 has been well publicized, with the hope for cures stretching beyond scientifically validated data. In particular, hydroxychloroquine and remdesivir are each undergoing a number of well-designed clinical trials (8), which are needed to accurately assess efficacy. In addition, scientific arguments are being put forward for a public-private partnership in preparing for the next possible coronavirus pandemic (9).

\section{Impact of COVID-19 on research} involving animal models

Research with animal models has been severely disrupted across the country. While the scope of the pandemic is unprecedented, most of the accredited institutions have benefited from the existence of emergency preparedness plans (Supple- 


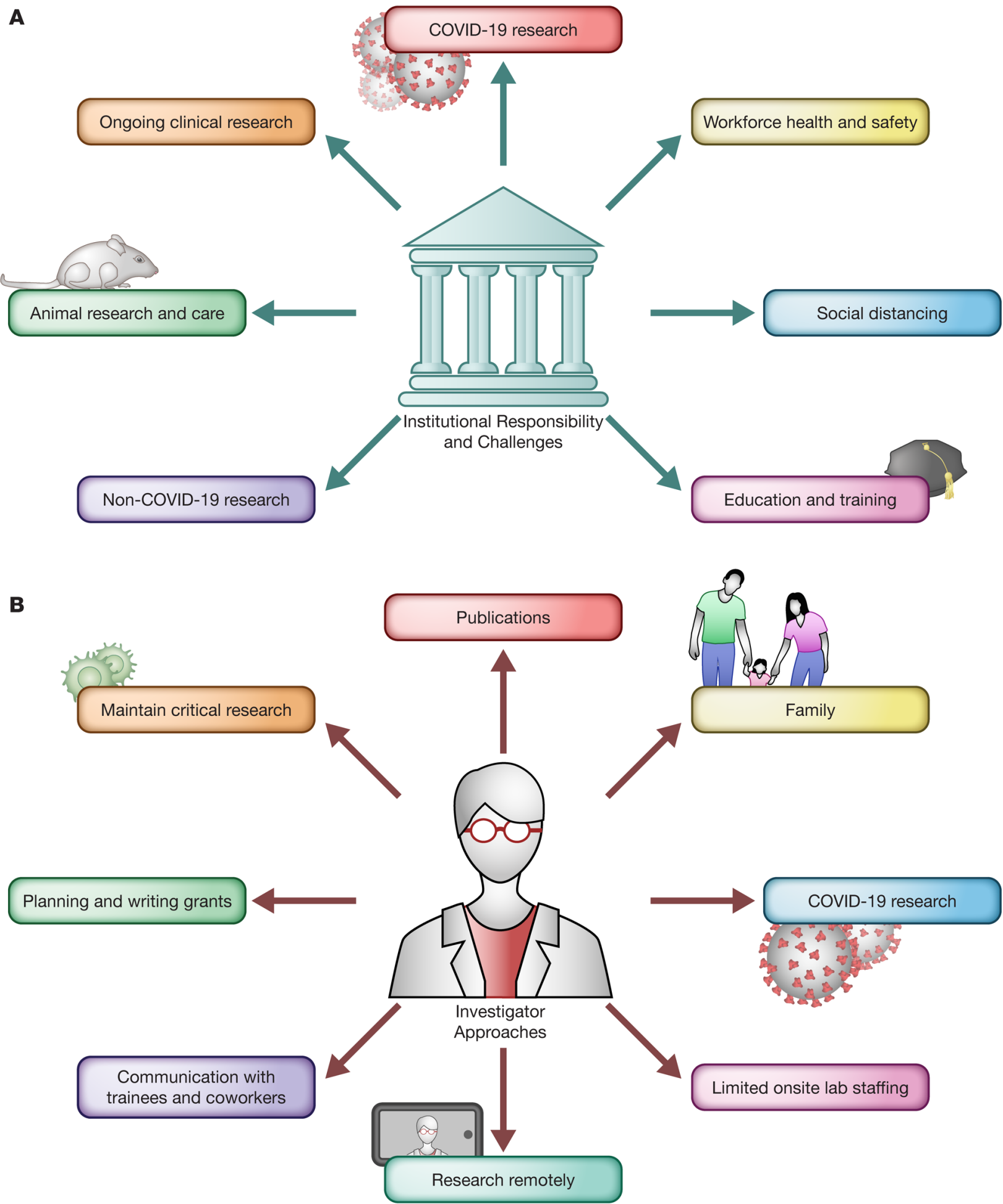

Figure 1. Impacts of the COVID-19 pandemic on research institutions and researchers. (A) COVID-19 poses new institutional responsibilities and challenges, including the need to ensure continuity of ongoing clinical research, increase COVID-19-related research, ensure safety of employees and students, enforce social distancing measures, ensure continuing the education of undergraduates and graduate students, restrict nonessential, non-COVID-19 research activities, and maintain required animal care resources. (B) Investigators have employed multiple approaches to cope with onsite lab restrictions and shutdowns. Researchers have maintained critical research activities, continued to submit publications, balanced work and family responsibilities, initiated or collaborated on COVID-19-related research, ensured limited onsite lab staffing, engaged in remote research opportunities, maintained regular and frequent communication with trainees and coworkers, and continued to plan and write research grants. Illustrated by Rachel Davidowitz. 
mental Table 2) and have reacted quickly to the challenge. Institutions have taken a phased approach to handling the crisis, with the main goal of safety of the workers, reducing the animal numbers, and supporting "critical" research activities. Steps that have been taken include stopping new animal orders (except for urgent needs), surgeries, and new experiments, but allowing time to complete ongoing studies. Restrictions have been placed on breeding beyond that required to maintain rare genetic lines. Researchers have been encouraged to utilize cryopreservation to preserve rodent strains. Changes to standard operating procedures to include longer cage-change intervals based on performance standards and reuse of PPE have been implemented. Securing the supply chain to ensure adequate food, bedding, enrichment, and chemicals for cage washing and disinfection are essential, and most vendors and suppliers of these functions have been able to meet the needs of the institutions. Animal care personnel have been classified as "essential personnel" and are utilizing split schedules, staggered shifts, and restricted hours in the vivarium to maintain social distancing needs and keep personnel safe. Enhanced sanitation in the common areas at the start and end of each shift is key to ensuring personnel safety. Communication in a timely manner between the animal care staff and research personnel is also critical to ensure smooth operation.

\section{How can we mobilize our efforts toward COVID-19-related research?}

In health care crises, baseline biometric measurements of an affected population are critical to truly determine the impact of a disaster on health. Lessons learned from previous health disasters, such as the Macondo oil spill, Hurricane Katrina, and Hurricane Sandy, for which such baseline measurements were unattainable, profoundly limited our research efforts. To gather baseline biometrics, an organization must nimbly leverage current clinical research assets and personnel to be deployed within hours or days. Platforms exist that can be mobilized quickly, such as hubs for Centers for Translational Science (CTSA) and National Cancer Institute-designated (NCI-designated) centers. The CTSA and NCI-designated hubs uniquely embrace a team-science approach and infrastructure to perform high quality research in streamlined and harmonized manners. The regulatory components of such hubs quickly engage IRBs and institutional procurement services to approve protocols and acquire the required materials and services to conduct research. For example, the New Jersey Alliance for Clinical and Translational Science (Rutgers CTSA Hub) conceptualized, obtained regulatory approval, and initiated recruitment within 12 days for a prospective, longitudinal study of health care workers exposed to SARS-CoV-2 in 750 participants. This study will quickly provide incidence and prevalence data in a vulnerable health care workforce. In tandem, the research team is performing appropriately powered randomized interventional controlled trials. Such emergent research programs can only be successful with extramural support. Accordingly, The Coronavirus Preparedness and Response Supplemental Appropriations Act signed into law March 6, 2020 (10) provides (through 2024) $\$ 8.3$ billion, of which $\$ 836$ million is directed to the NIH, $\$ 61$ million to the FDA, \$950 million to the CDC, and $\$ 2$ billion for the Biomedical Advanced Research and Development Authority. The NIH and CDC have already announced several requests for applications (RFAs) (Supplemental Table 3). Although the appropriations will undoubtedly aid in performing quality and impactful research at a time of crisis, the initial clinical research, protocol generation, and regulatory efforts must occur nimbly with limited current institutional and extramural support. Approaches to quickly deploy federal and state extramural support to gather baseline biometrics will ensure improved health outcomes for Americans devastated by health crises.

\section{How can research personnel be safe and productive?}

While fully grasping the gravity of the pandemic, major frustrations of researchers include feeling unproductive and unable to advance potentially exciting discoveries, and being homebound and less active. If there is any silver lining, it's knowing that our nation will overcome the impasse while doing the right thing to prevent the spread of infection. It has been gratifying to see how investigators and trainees have embraced the challenge in complementary ways (Figure 1B). Communication remains effective via various video teleconferencing modalities, albeit not entirely replacing the benefits of face-to-face meetings. Clinical research not involving patient contact can be maintained remotely, as can many patient encounters.

Several ways to maintain research staff engagement, while working remotely, have been presented (11). There is more time now than ever to plan and write grants, write that long-contemplated review article, and submit papers even if they don't have the biologic mechanism completely worked out. We may need to compromise on the target journal in order to maintain research momentum and fulfill our trainee needs. The next generation of researchers will carry this crisis with them for their entire careers and will be shaped by the environment and opportunities we create for them today. Preparing applications for postdoctoral, e.g., K-type (12) or society/foundation awards, and predoctoral career development awards is important to consider (albeit regardless of COVID-19). Virtual lab meetings, research seminars, and "chalk talks" with colleagues can be readily arranged, with additional potential contact time with lab members via assigned journal clubs. There are also tremendous funding opportunities in COVID-19-related research (Supplemental Table 3); hence, looking for collaborations outside our comfort zone can be beneficial on several levels. Although virologists are best suited to work on SARS-CoV-2, this is also the ideal time to engage (and vice versa) immunologists, computational, modeling, and artificial intelligence experts, chemists, drug developers, engineers, noninfectious disease clinical investigators, and intensivists, among other disciplines.

It is also time to spend with family and friends. Each day over the past many weeks, the media has reported on creative ways to celebrate those special events birthdays, anniversaries, weddings, births, etc. This human connection is critical to our physical, mental, and professional well-being. This crisis will end, and we will emerge ready to embrace a brighter future. 


\section{Acknowledgments}

The authors are grateful to the assistance of Marika Dunn for collecting some of the presented information, and Neil Grant for preparing the figure and supplemental tables. This Viewpoint salutes all the health care workers who are at the front lines of the COVID-19 pandemic, helping patients and their families.

Address correspondence to: M. Bishr Omary, Center for Advanced Biotechnology and Medicine, Rutgers University, 679 Hoes Lane West, Piscataway, New Jersey 08854, USA.Email: bishr.omary@rutgers.edu.

1. CDC. Influenza (Flu). Past pandemics. 1918 Pandemic (H1N1 virus). http://www.cdc.gov/ flu/pandemic-resources/1918-pandemich1n1.html. Updated March 20, 2019. Accessed April 7, 2020.

2. Research Collaboratory for Structural Bioinformatics. Protein Data Bank. 6LU7. http://www.
rcsb.org/structure/6LU7. Updated March 18, 2020. Accessed April 7, 2020.

3. Hayley Slusser. Rutgers researchers develop test for coronavirus to expand access in NJ. Daily Targum. April 1, 2020. http://www.dailytargum. com/article/2020/04/rutgers-researchersdevelop-test-for-coronavirus-to-expand-accessin-nj. Accessed April 7, 2020.

4. Darley J. Face shield designs to fill the gap. Delve. March 19, 2020. http://www.delve.com/ insights/face-shield-designs-to-fill-the-gap. Accessed April 7, 2020.

5. Rutgers, the State University of New Jersey. Rutgers New Jersey Medical School research team participates in first external evaluation of groundbreaking COVID-19 test. March 21, 2020. http://academichealth.rutgers.edu/ news/2020-03-21-rutgers-new-jersey-medicalschool-research-team-participates-first-external. Accessed April 7, 2020.

6. Cortez, MC. Abbott launches 5-minute virus test for use almost anywhere. Bloomberg. March 27, 2020. http://www.bloomberg.com/news/ articles/2020-03-27/abbott-launches-5-minutecovid-19-test-for-use-almost-anywhere. Updated March 28, 2020. Accessed April 7, 2020.
7. Austin J. COVID-19 clinical trials launch at U of M. KARE 11. March 18, 2020. http://www.kare11. com/article/news/local/covid19-clinical-trialslaunch-at-u-of-m/89-e81f214f-2df5-4e09-beca46d4c94c741c. Accessed April 7, 2020.

8. ClinicalTrials.gov. COVID-19. http://www. clinicaltrials.gov/ct2/results?cond=COVID-19. Accessed April 7, 2020.

9. Burley SK. How to help the free market fight coronavirus. Nature. 2020;580(7802):167.

10. Lowey, NM. H.R.6074 - Coronavirus Preparedness and Response Supplemental Appropriations Act, 2020. http://www.congress.gov/ bill/116th-congress/house-bill/6074. Updated March 6, 2020. Accessed Aprl 7, 2020.

11. University of Michigan Medical School Office of Research. Potential activities for research staff during the assignment to remote work. http://research.medicine.umich.edu/potentialactivities-research-staff-during-assignmentremote-work. Updated March 27, 2020. Accessed April 7, 2020.

12. Conte ML, Omary MB. NIH Career Development Awards: conversion to research grants and regional distribution. J Clin Invest. 2018;128(12):5187-5190. 